\title{
Pulmonary and right ventricular dysfunction are frequently present in heart failure irrespective of left ventricular ejection fraction
}

\author{
Wouter Robaeys, ${ }^{1,2}$ Sema Bektas, ${ }^{1}$ Josiane Boyne, ${ }^{1}$ Vanessa van Empel, ${ }^{1}$ \\ Nicole Uszko-Lencer, ${ }^{1,3}$ Christian Knackstedt, ${ }^{1}$ Hans-Peter Brunner-La Rocca ${ }^{1}$
}

'Department of Cardiology, Maastricht University Medical Center, Maastricht, The

Netherlands

${ }^{2}$ Cardiovascular Research Institute Maastricht (CARIM), Maastricht, The Netherlands ${ }^{3}$ Department of Research and Education, Center of Expertise for Chronic Organ Failure (CIRO+), Horn, The Netherlands

\section{Correspondence to} Dr Wouter Robaeys, Department of Cardiology, Maastricht University Medical Center (MUMC), 6202 AZ Maastricht 5800, The Netherlands; wouter. robaeys@mumc.nl

WR and SB contributed equally.

Received 3 April 2017

Revised 7 July 2017

Accepted 11 July 2017

\section{ABSTRACT \\ Background Heart failure (HF) may influence the} lungs and vice versa. However, this interaction and the influence on right ventricular function (RVF) are insufficiently described in patients with HF divided into the recent groups based on left ventricular ejection fraction (LVEF): HF with reduced, midrange and preserved ejection fraction (HFrEF, HFmrEF and HFpEF, respectively). Methods Overall, 186 consecutive stable patients with HF seen in our outpatient clinic were retrospectively divided into HFrEF $(n=70)$, HFmrEF ( $n=55)$ and HFpEF $(n=61)$. Airflow limitation and gas exchange disturbance were measured by spirometry (forced expiratory volume in the first second/forced vital capacity (FEV /FVC) (\%)) and diffusion capacity of the lungs for carbon monoxide (DLCO). Standard echocardiography was performed to measure RV structure (RV diameter) and function (tricuspid annular plane systolic excursion/pulmonary artery systolic pressure (TAPSE/PASP)). Correlations were used to assess possible relations between pulmonary dysfunction and measurements of the RV.

Results None of the investigated parameters differed significantly between the three groups (all $p>0.1$ ); FEV I FVC was $70 \% \pm 12 \%, 70 \% \pm 13 \%$ and $74 \% \pm 10 \%$ in patients with HFrEF, HFmrEF and HFpEF $(p=0.12)$ and DLCO was $5.7 \pm 1.6,5.7 \pm 1.8$ and $5.6 \pm 1.6 \mathrm{mmol} / \mathrm{min} /$ $\mathrm{kPa}$, respectively $(\mathrm{p}=0.95)$. RV structure and function did not differ either (TAPSE/PASP 0.58, 0.60 and 0.57, respectively $(p=0.84))$. There was a correlation of DLCO with RV function $(r=0.34, p<0.001)$.

Conclusion The investigated cardiopulmonary parameters were comparable in the three HF groups. Diffusion capacity was impaired in more than half of the stable HF population independently of the LVEF and showed a correlation with RV function.

\section{INTRODUCTION}

Heart Failure (HF) used to be subdivided into 'HF with preserved Ejection Fraction' (HFpEF) and 'HF with reduced Ejection Fraction' (HFrEF). This segmentation is based on the left ventricular ejection fraction (LVEF) being higher or lower than 40\%-50\%. Recently, HF with midrange EF (HFmrEF) was added as a new category representing a LVEF ranging from $40 \%$ to $49 \%$ to better explore this so far largely neglected subpopulation. ${ }^{1}$

In patients with $\mathrm{HF}$, obstructive lung function abnormalities and disturbance in gas exchange are common. $^{2}{ }^{3}$ Both, chronic obstructive pulmonary disease (COPD) and low diffusion capacity, are associated with a worse prognosis. ${ }^{45}$ Nevertheless, the diagnosis of COPD and low diffusion capacity in patients with HF is challenging. Thus, measures of airflow limitation (AL) and diffusion capacity of the lungs for carbon monoxide (DLCO) are only reliable in a stable stage of $\mathrm{HF}^{6}$ Still, research regarding the presence of lung function abnormalities has been done often in unstable HF patients. ${ }^{7}$

Precipitating factors contributing to HF hospitalisation in the three different HF groups were recently explored. ${ }^{8}$ Pneumonia was found to be an important factor in all groups, suggesting that the lungs play an important role in HF patients irrespectively of LVEF. However, information related to pulmonary function is lacking in the three $\mathrm{HF}$ groups. Thus, although routine testing of AL and DLCO may be relevant in all stable patients with $\mathrm{HF}$, this has not yet been investigated in three different HF groups to explore if such abnormalities are particularly prevalent in one of these groups.

Vice versa, COPD may influence the heart as well, for example, by causing pulmonary hypertension. ${ }^{9}$ A study investigating patients with COPD did not show clear functional changes in the right heart. ${ }^{3}$ In this study, the right ventricular (RV) function was estimated through tricuspid annular plane systolic excursion (TAPSE), which was normal in this and another population. ${ }^{9}$ Nevertheless, the ratio TAPSE to pulmonary artery systolic pressure (PASP) may be better suited to assess RV function than each of the two variables alone. ${ }^{10}$ Moreover, TAPSE/PASP may be prognostically important in $\mathrm{HF}^{11}$ but has not yet been studied in a stable HF population subdivided into the three different HF groups.

Therefore, the primary aim of this study was to investigate pulmonary function (i.e., $\mathrm{AL}$ and disturbance in gas exchange) and RV function (i.e., TAPSE/PASP) in a stable HF population divided into the three recently defined groups based on LVEF (i.e., HFpEF, HFmrEF and HFrEF). In addition, we investigated the association between cardiopulmonary tests in all patients with HF to explore the potential interplay between pulmonary functional abnormalities and HF on both organs.

\section{METHODS}

\section{Study design and participants}

Between October 2012 and November 2013, consecutive patients visiting the outpatient HF clinic at Maastricht University Medical Center (The Netherlands) were screened for inclusion in this prospective cross-sectional observational study. The purpose of this study was to perform a cohort study 
until a total of 200 patients were included. Patients were eligible for inclusion if they fulfilled the following criteria: a recent diagnosis of HF based on current guidelines, ${ }^{1} 12$ age above 50 years and clinically stable conditions for at least 1 month. The diagnosis for inclusion was based on criteria of the European Society of Cardiology (ESC) 2012. ${ }^{12}$ The separation into the three groups related to LVEF was based on the ESC guidelines 2016. All patients also fulfilled the criteria of HF based on the ESC guidelines 2016. Patients were selected irrespective of LVEF. Patients were excluded if: the measurement of LVEF was lacking, patients were not able to cooperate or when spirometry was clinically contraindicated (detached retina, active tuberculosis and resting pulse $>120 / \mathrm{min})$. Other exclusion criteria included recent surgery, myocardial infarction ( $<1$ month), uncorrected severe valvular disease, lower respiratory tract infection or pneumothorax within the last 2 months and a stroke within the last 12 months. Overall, 205 patients with HF volunteered to participate in this study. Nineteen patients were excluded from further analysis due to missing of recent echocardiography; thus, 186 patients with the diagnosis of HF were eligible for the present study. Patients not included did not differ significantly from those included regarding their baseline characteristics (data not shown). Written informed consent was provided by all patients. This study complies with the Declaration of Helsinki. The local medical ethics committee approved this study (METC 12-2-005 (NL39223.068.12)).

\section{Measurements and data collection \\ Clinical characteristics}

The following patients characteristics were recorded as clinically available: age, gender, HF aetiology, comorbidities, cardiovascular risk factors (diabetes, hypertension, hypercholesterolaemia and smoking), New York Heart Association Functional Classification (NYHA-class), blood pressure, heart rate, (non-invasive) $\mathrm{O}$-saturation at rest, $\mathrm{N}$-terminal probrain natriuretic peptide (NT-proBNP), electrocardiography and current medication.

\section{Pulmonary assessment}

Lung function was measured before and after administration of $200 \mu \mathrm{g}$ salbutamol. Spirometry (Masterscreen, Jaeger, Würzburg, Germany) was performed by trained respiratory technicians in accordance with the recommendations from the European Respiratory Society (ERS). ${ }^{13}$ The Global initiative for Obstructive Lung Disease (GOLD) criteria were used to define $\mathrm{AL}$ in COPD using the Tiffeneau Index (i.e., forced expiratory volume in the 1 st second $\left(\mathrm{FEV}_{1}\right) /$ forced vital capacity $\left.(\mathrm{FVC}) \%\right)$. Thus, patients were diagnosed with COPD if $\mathrm{FEV}_{1} / \mathrm{FVC}$ after bronchodilatation was lower than $70 \%$. Furthermore, the severity of AL was staged according to the GOLD criteria. An AL was considered to be mild, moderate, severe and very severe if the predicted percentage of $\mathrm{FEV}_{1}$ was $\geq 80 \%, 50 \%-79 \%, 30 \%-49 \%$ or $<30 \%$, respectively. ${ }^{14}$ Patients fulfilling the diagnostic criteria for COPD were subsequently stratified according to symptoms and future risk. ${ }^{14}$ DLCO is an individual variable based on the patient's length, age and gender and is measured according to

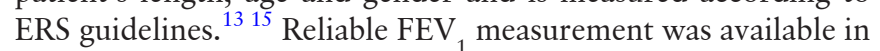
all but two patients. Twelve patients did not comply sufficiently to reliably assess of DLCO.

\section{Echocardiography}

A prerequisite for study inclusion was a comprehensive standard echocardiographic examination within a maximum of 6 months prior to enrolment in the study. ${ }^{16}$ If a recent cardiac echocardiography was not available, it was repeated for this study. However, in 19 cases, patients refrained from repeating it. These cases were excluded from the study. The following parameters were assessed: LVEF, RV diameter (RVD), RV function (TAPSE/PASP), $\mathrm{E} / \mathrm{e}$ ' and left atrium volume index (LAVI). In case of absence of regional differences in contractility, M-mode (Teichholz) was used to determine LVEF. If contractility was not uniform for all segments of the LV, biplane Simpson method was used (apical 4-chamber and 2-chamber view). In order to determine LAVI, biplane method from 4-chamber and 2-chamber apical view was used prior to opening of mitral valve and volumes were divided by body surface area. HF was divided into three groups according to recent guidelines based on LVEF: HFrEF, HFmrEF and HFpEF. ${ }^{1}$

\section{Statistical analysis}

Statistical analysis was performed with the use of IBM SPSS Statistics software V.23. Descriptive data are presented as mean $\pm S D$, frequencies (\%) or median (IQR) as appropriate. Continuous variables with a normal distribution are compared with the use of analysis of variance test. Continuous variables with a non-normal distribution are compared using the Kruskal-Wallis test. Categorical variables are compared with the use of $\chi^{2}$ test or Fisher's exact tests, as appropriate. p Values of less than 0.05 are considered to indicate statistical significance. Multiple comparisons are adjusted applying post hoc Bonferroni test. To determine the association between lung function abnormalities and cardiac function, Pearson or Spearman rank correlations are used, as appropriate. Based on the power of this relatively small study population, no multivariable regression analysis was performed on this study population to investigate potential predisposing factors for cardiopulmonary abnormalities (i.e., AL, DLCO and TAPSE/PASP).

\section{RESULTS}

\section{Patient characteristics}

The baseline characteristics of the three groups based on LVEF are given in table 1 . The mean age was $75 \pm 10$ years, and the majority of the patients were male. The median LVEF was 44 [32-53]\%.

The most common cause of HF was coronary artery disease in the HFrEF and HFmrEF groups and hypertensive heart disease in the HFpEF group. Cardiac risk factors were similar in all three HF groups. Approximately $80 \%$ of the patients had no or little symptoms during daily life activities (i.e., NYHA classes I and II). The NT-proBNP level in the HFpEF patient group was significant lower compared with HFrEF $(p=0.01)$ (table 1$)$.

\section{Spirometry}

No significant differences in AL or DLCO were observed between the three groups (table 2). On average, the mean Tiffeneau index was close to the cut-off of being abnormal and approximately half of all patients with HF had abnormal DLCO values. The percentage of predicted DLCO was overall slightly reduced with no significant differences between the three groups. Not unexpectedly, the percentage of predicted DLCO in HF patients with and without AL, respectively, differed significantly $(p<0.001)$ but remained significantly reduced in both groups. There were no significant differences between the three groups based on LVEF in both, patients with and without AL (table 2).

\section{Echocardiography}

The echocardiographic findings are given in table 3. Measures of RVD and RV function (i.e., TAPSE/PASP) were similar in patients with HFrEF, HFmrEF and HFpEF. The diameter of the RV was 
Table 1 Baseline characteristics in an HF population divided into three groups

\begin{tabular}{|c|c|c|c|c|c|c|c|c|}
\hline \multirow[b]{2}{*}{ Demographics } & \multirow[b]{2}{*}{ Total } & \multicolumn{3}{|c|}{ Ejection fraction (\%) } & \multicolumn{4}{|l|}{$\mathrm{p}$} \\
\hline & & $\begin{array}{l}\text { HFrEF } \\
\text { LVEF: } \\
<40 \\
(n=70)\end{array}$ & $\begin{array}{l}\text { HFmrEF } \\
\text { LVEF: } \\
40-49 \\
(n=55)\end{array}$ & $\begin{array}{l}\text { HFpEF } \\
\text { LVEF: } \\
\geq 50 \\
(n=61)\end{array}$ & Total & $\begin{array}{l}\text { HFrEF } \\
\text { versus } \\
\text { HFmrEF }\end{array}$ & $\begin{array}{l}\text { HFmrEF } \\
\text { versus } \\
\text { HFpEF }\end{array}$ & $\begin{array}{l}\text { HFrEF } \\
\text { versus } \\
\text { HFpEF }\end{array}$ \\
\hline Age (years) & $75 \pm 10$ & $73 \pm 10$ & $75 \pm 10$ & $77 \pm 9$ & 0.17 & $>0.999$ & 0.95 & 0.18 \\
\hline $\begin{array}{l}\text { Male (gender) } \\
\mathrm{n}(\%)\end{array}$ & $125(67)$ & $53(76)$ & $42(76)$ & $30(49)$ & $0.001 *$ & $>0.999$ & 0.01 * & 0.01 * \\
\hline DCM & $34(18)$ & $18(26)$ & $9(16)$ & $7(12)$ & - & - & - & - \\
\hline $\mathrm{HHD}$ & $35(19)$ & $2(3)$ & $7(13)$ & $26(43)$ & - & - & - & - \\
\hline VHD & $10(5)$ & $4(6)$ & $1(2)$ & $5(8)$ & - & - & - & - \\
\hline \multicolumn{9}{|l|}{ Comorbidity (n (\%)) } \\
\hline DM & $53(29)$ & 27 (39) & $11(20)$ & $15(25)$ & 0.053 & 0.09 & $>0.999$ & 0.27 \\
\hline I and II & $18(13)$ & $8(16)$ & $6(14)$ & $4(8)$ & 0.23 & $>0.999$ & 0.63 & 0.30 \\
\hline III and IV & $7(5)$ & $4(8)$ & $3(7)$ & 0 & & & & \\
\hline No history COPD & $114(80)$ & $40(77)$ & $35(80)$ & $39(85)$ & & & & \\
\hline \multicolumn{9}{|l|}{ Smoking history $(n=178)(n(\%))$} \\
\hline No smoker & $53(30)$ & $16(23)$ & $14(29)$ & $23(38)$ & 0.30 & $>0.999$ & 0.87 & 0.51 \\
\hline Current smoker & $22(12)$ & $10(15)$ & $4(8)$ & $8(13)$ & & & & \\
\hline Former smoker & $103(58)$ & $43(62)$ & $31(63)$ & $29(48)$ & & & & \\
\hline \multicolumn{9}{|l|}{ Signs and symptoms } \\
\hline \multirow[t]{2}{*}{ NYHA $(n(\%))$} & $145(78)$ & $57(81)$ & $44(80)$ & $44(72)$ & 0.40 & $>0.999$ & 0.96 & 0.63 \\
\hline & $41(22)$ & $13(19)$ & $11(20)$ & $17(28)$ & & & & \\
\hline \multirow{3}{*}{$\begin{array}{l}\text { Sinus rhythm } \\
\text { Atrial Fibrillation } \\
\text { Other }\end{array}$} & $116(62)$ & $46(66)$ & $36(66$ & $34(56)$ & 0.06 & 0.06 & $>0.999$ & 0.15 \\
\hline & $34(18)$ & $6(9)$ & $13(24$ & $15(25)$ & & & & \\
\hline & $35(19)$ & $18(26)$ & $6(11$ & $11(18)$ & & & & \\
\hline \multicolumn{9}{|l|}{ Cardiac medications (n (\%)) } \\
\hline ACE-I & $166(89)$ & 66 (94) & $51(93)$ & $49(80)$ & $0.02^{*}$ & $>0.999$ & 0.18 & 0.06 \\
\hline Beta blocker & $171(92)$ & $68(97)$ & $48(87)$ & $55(90)$ & 0.11 & 0.12 & $>0.999$ & 0.45 \\
\hline Diuretics & $147(79)$ & $60(86)$ & $43(78)$ & $44(72)$ & 0.16 & $>0.999$ & $>0.999$ & 0.24 \\
\hline Aldosterone antagonist & 70 (38) & $37(53)$ & $22(40)$ & $11(18)$ & & 0.63 & $0.03^{*}$ & $0.003^{*}$ \\
\hline \multicolumn{9}{|c|}{$\begin{array}{l}\text { Mean }( \pm S D) \text {. } \\
\text { Median (IQR). } \\
\text { p Values below } 0.05 \text { in bold and with asterisks. } \\
\text { ACE-I, Angiotensin Converting Enzyme Inhibitor; BP, blood pressure; bpm, beats per minute; } C A D \text {, coronary artery disease; COPD, chronic obstructive pulmonary disease; DCM, dilated } \\
\text { cardiomyopathy; DM, diabetes mellitus; GOLD, Global initiative for Obstructive Lung Disease; HC, hypercholesterolaemia; HF, heart failure; HFmrEF, heart failure midrange ejection fraction; } \\
\text { HFpEF, heart failure preserved ejection fraction; HFrEF, heart failure reduced ejection fraction; HHD, hypertensive heart disease; HT, hypertension; LVEF, left ventricular ejection fraction; NT-- } \\
\text { proBNP, N-terminal probrain natriuretic peptide; NYHA, New York Heart Association; } O, \text { oxygen; VHD, valvular heart disease. }\end{array}$} \\
\hline
\end{tabular}

enlarged in $30 \%$, and the RV function was decreased in $27 \%$ of all patients with HF. Furthermore, parameters of diastolic function (i.e., LAVI, E/e') were similar in the three groups based on LVEF (table 3).

\section{Correlations}

The correlations between RV function and pulmonary dysfunctions are given in table 4. In general, the correlations were relatively weak.
FEV /FVC (\%) showed a negative correlation with TAPSE/ PASP. DLCO was positively correlated to the TAPSE/PASP and RV diameter and negatively correlated to PASP.

TAPSE/PASP showed negative correlations with RVD and NT-ProBNP. PASP was also positively correlated with RVD and NT-proBNP. (table 4). No correlations are found between TAPSE and DLCO, AL and PASP or between AL and PASP, NT-ProBNP. 


\section{Original research}

Table 2 Spirometry findings in an HF population divided into three groups

\begin{tabular}{|c|c|c|c|c|c|c|c|c|}
\hline \multirow[b]{2}{*}{ Spirometry } & \multirow[b]{2}{*}{ Total } & \multirow{2}{*}{$\begin{array}{l}\text { HFrEF } \\
\text { LVEF: } \\
<40 \\
(n=70)\end{array}$} & \multirow{2}{*}{$\begin{array}{l}\text { HFmrEF } \\
\text { LVEF: } \\
40-49 \\
(n=55)\end{array}$} & \multirow{2}{*}{$\begin{array}{l}\text { HFpEF } \\
\text { LVEF: } \\
\geq 50 \\
(n=61)\end{array}$} & \multicolumn{4}{|l|}{ p Value } \\
\hline & & & & & Total & $\begin{array}{l}\text { HFrEF } \\
\text { versus } \\
\text { HFmrEF }\end{array}$ & $\begin{array}{l}\text { HFmrEF } \\
\text { versus } \\
\text { HFpEF }\end{array}$ & $\begin{array}{l}\text { HFrEF } \\
\text { versus } \\
\text { HFpEF }\end{array}$ \\
\hline Diagnosis COPD $(n=186)(n(\%))$ & $62(33)$ & $26(37)$ & $20(36)$ & $16(26)$ & 0.70 & $>0.99$ & $>0.99$ & $>0.99$ \\
\hline $\mathrm{FEV}_{1}(\mathrm{~L})(\mathrm{n}=184)$ & $2.3 \pm 0.7$ & $2.4 \pm 0.7$ & $2.4 \pm 0.8$ & $2.3 \pm 0.7$ & 0.68 & $>0.99$ & $>0.99$ & $>0.99$ \\
\hline FVC $(L)(n=184)$ & $3.3 \pm 0.9$ & $3.4 \pm 0.9$ & $3.4 \pm 0.9$ & $3.1 \pm 1.0$ & 0.14 & $>0.99$ & 0.17 & 0.41 \\
\hline $\mathrm{FEV}_{1} / \mathrm{FVC}(\%)(\mathrm{n}=184)$ & $71.2 \pm 11.7$ & $70.2 \pm 11.9$ & $69.7 \pm 13.2$ & $73.8 \pm 9.7$ & 0.12 & $>0.99$ & 0.20 & 0.27 \\
\hline $\mathrm{FEV}_{1} \%$ pred $(\mathrm{n}=175)$ & $91.3 \pm 21.2$ & $82.1 \pm 20.5$ & $86.3 \pm 19.8$ & $94.0 \pm 21.8$ & 0.01 * & 0.81 & 0.15 & $<0.01 *$ \\
\hline $\mathrm{DLCO}(\mathrm{mmol} / \mathrm{min} / \mathrm{kPa})(\mathrm{n}=174)$ & $5.6 \pm 1.7$ & $5.7 \pm 1.6$ & $5.7 \pm 1.8$ & $5.6 \pm 1.6$ & 0.95 & $>0.99$ & $>0.99$ & $>0.99$ \\
\hline DLCO \% pred $(n=174)$ & $69.1 \pm 16.0$ & $67.6 \pm 14.4$ & $67.9 \pm 19.1$ & $72.5 \pm 15.4$ & 0.20 & $>0.99$ & 0.42 & 0.30 \\
\hline Abnormal DLCO (n=141) (n (\%)) & $77(55)$ & $29(59)$ & $26(55)$ & $22(49)$ & 0.60 & $>0.99$ & $>0.99$ & 0.96 \\
\hline Abnormal DLCO in pts without AL $(n=77)$ & $39(51)$ & $14(58)$ & $12(48)$ & $13(46)$ & 0.66 & 0.47 & 0.91 & 0.39 \\
\hline DLCO \% pred in pts with $A L(n=61)$ & $63.5 \pm 16.1$ & $65.5 \pm 14.2$ & $56.8 \pm 19.7$ & $68.8 \pm 11.2$ & 0.06 & 0.20 & 0.07 & $>0.99$ \\
\hline DLCO \% pred in pts without AL $(n=98)$ & $74.2 \pm 15.3$ & $70.7 \pm 13.8$ & $75.9 \pm 16.3$ & $76.3 \pm 15.6$ & 0.24 & 0.54 & $>0.99$ & 0.38 \\
\hline
\end{tabular}

Mean ( \pm SD).

Median (IQR).

$\mathrm{p}$ Values below 0.05 in bold and with asterisks.

COPD, chronic obstructive pulmonary disease; DLCO, diffusing lung capacity for carbon monoxide; FEV , forced expiratory volume in $1 \mathrm{~s}$; FVC, forced vital capacity; HF, heart failure; HFmrEF,

heart failure midrange ejection fraction; HFpEF, heart failure preserved ejection fraction; HFrEF, heart failure reduced ejection fraction; pred, predicted.

\section{DISCUSSION}

This study investigated to what extent lung function abnormalities and RV dysfunction might depend on LVEF. Lung function abnormalities and reduced RV function were common in this outpatient
HF population without any differences between the three groups based on EF. Furthermore, a weak association between pulmonary and RV function was found across all HF groups, suggesting a limited influence of lung function abnormalities on the RV.

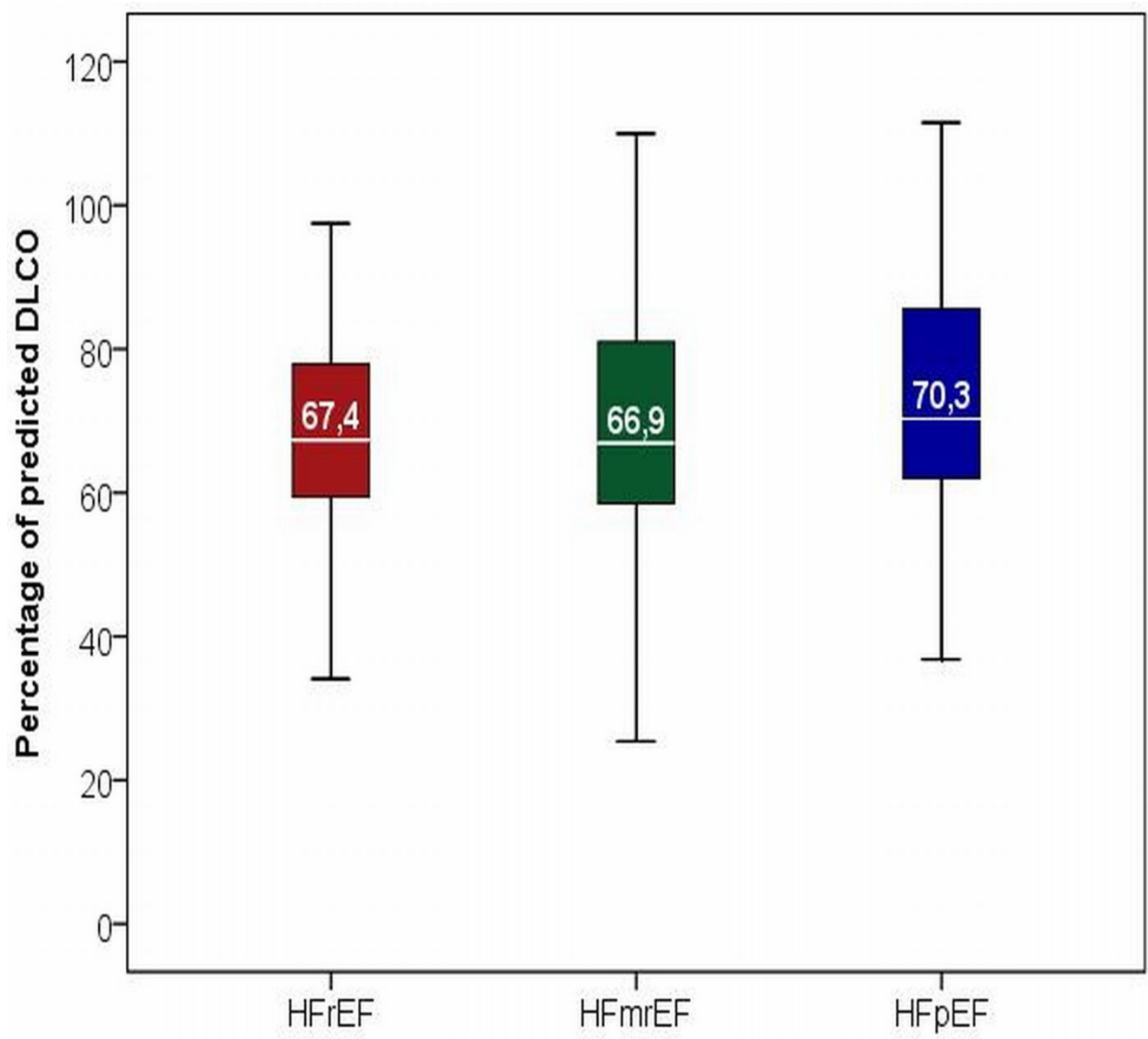

\section{HF groups}

Figure 1 Percentage of predicted DLCO in three groups based on LVEF. DLCO, diffusion capacity of the lungs for carbon monoxide; HF, heart failure; $\mathrm{HFmrEF}$, heart failure midrange ejection fraction; HFpEF, heart failure preserved ejection fraction; HFrEF, heart failure reduced ejection fraction; LVEF, left ventricular ejection fraction. 


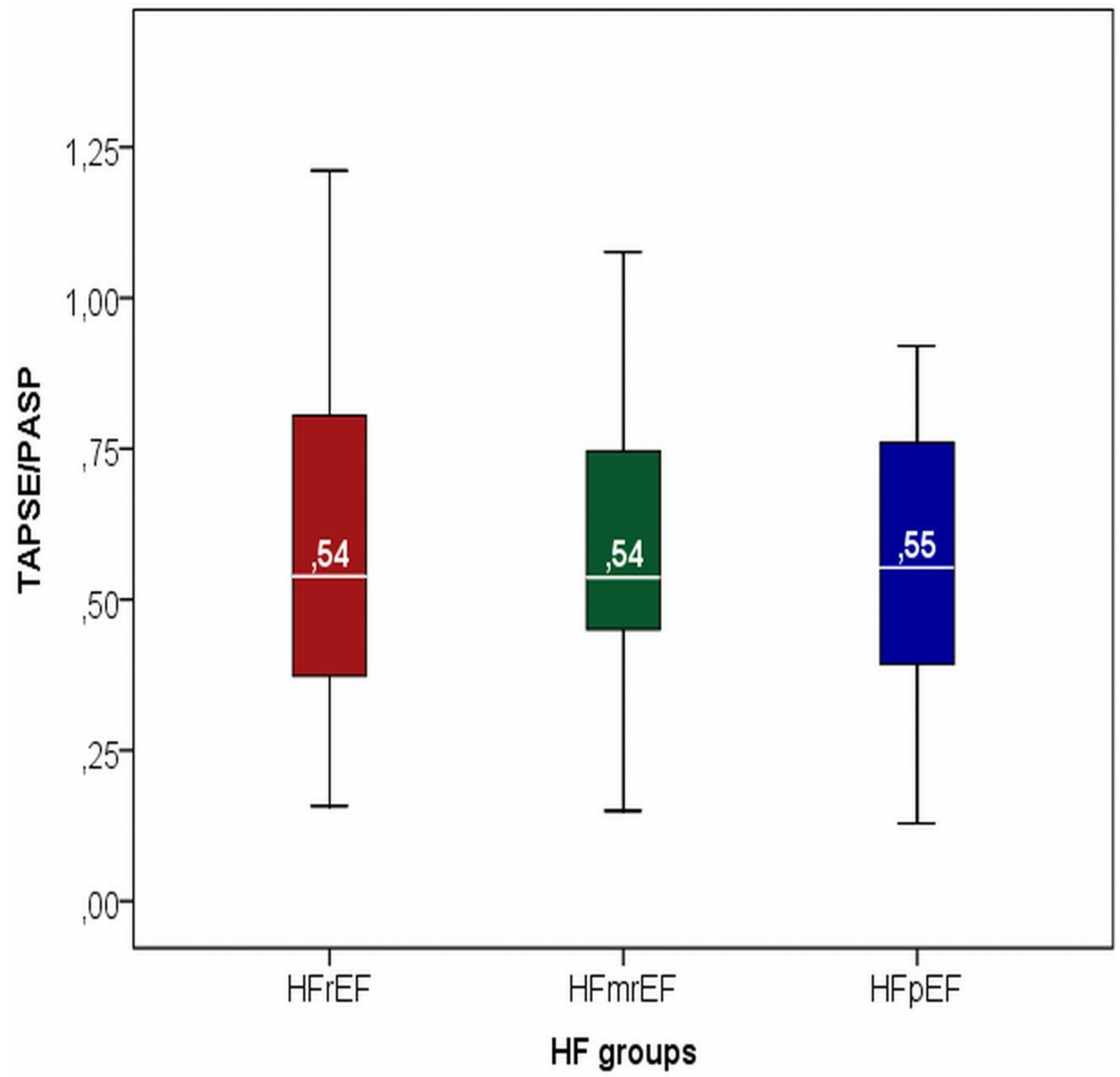

Figure 2 TAPSE/PASP in three groups based on LVEF. HF, heart failure; HFmrEF, gheart failure midrange ejection fraction; HFpEF, heart failure preserved ejection fraction; HFrEF, heart failure reduced ejection fraction; LVEF, left ventricular ejection fraction; PASP, pulmonary artery systolic pressure; TASPE, tricuspid annular plane systolic excursion.

Table 3 Echocardiography findings in an HF population divided into three groups

\begin{tabular}{|c|c|c|c|c|c|c|c|c|}
\hline \multirow[b]{2}{*}{ Echocardiography } & \multirow[b]{2}{*}{ Total } & \multirow{2}{*}{$\begin{array}{l}\text { HFrEF } \\
\text { LVEF: } \\
<40 \\
(n=70)\end{array}$} & \multirow{2}{*}{$\begin{array}{l}\text { HFmrEF } \\
\text { LVEF: } \\
40-49 \\
(n=55)\end{array}$} & \multirow{2}{*}{$\begin{array}{l}\text { HFpEF } \\
\text { LVEF: } \\
\geq 50 \\
(n=61)\end{array}$} & \multicolumn{4}{|l|}{$p$ Values } \\
\hline & & & & & Total & $\begin{array}{l}\text { HFrEF } \\
\text { versus } \\
\text { HFmrEF }\end{array}$ & $\begin{array}{l}\text { HFmrEF } \\
\text { versus } \\
\text { HFpEF }\end{array}$ & $\begin{array}{l}\text { HFrEF } \\
\text { versus } \\
\text { HFpEF }\end{array}$ \\
\hline $\operatorname{LVEF}(\%)(n=186)$ & $44(32-53)$ & $29(24-34)$ & $45(43-48)$ & $58(53-62)$ & $<0.001^{*}$ & $<0.001^{*}$ & $<0.001^{*}$ & $<0.001 *$ \\
\hline $\operatorname{RVD}(m m)(n=174)$ & $40 \pm 7$ & $39 \pm 7$ & $40 \pm 6$ & $42 \pm 7$ & 0.09 & $>0.999$ & 0.72 & 0.08 \\
\hline Abnormal RVD (n (\%)) & $56(30)$ & $19(27)$ & $17(31)$ & $20(33)$ & 0.77 & 0.64 & 0.83 & 0.48 \\
\hline TAPSE/PASP $(\mathrm{mm} / \mathrm{mm} \mathrm{Hg})(\mathrm{n}=138)$ & $0.58 \pm 0.25$ & $0.59 \pm 0.27$ & $0.60 \pm 0.26$ & $0.57 \pm 0.22$ & 0.84 & $>0.999$ & $>0.999$ & $>0.999$ \\
\hline $\begin{array}{l}\text { Abnormal TAPSE/PASP }(<40 \mathrm{~mm} / \mathrm{mm} \mathrm{Hg}) \\
(n=138)(\mathrm{n}(\%))\end{array}$ & $37(27)$ & $16(33)$ & $8(19)$ & $13(28)$ & 0.31 & 0.13 & 0.28 & 0.64 \\
\hline E/e' (mean septal and lateral) $(n=132)$ & $10.0(7.9-14.9)$ & $10.8(6.9-18.0)$ & $9.9(8.3-14.5)$ & $9.8(7.9-14.0)$ & 0.73 & $>0.999$ & $>0.999$ & $>0.999$ \\
\hline $\begin{array}{l}\text { LAVI (LAV/BSA) }(n=167) \\
\text { (biplane method) }\end{array}$ & $43(36-55)$ & $47(39-55)$ & $40(31-53)$ & $44(35-57)$ & 0.09 & 0.09 & 0.72 & $>0.999$ \\
\hline
\end{tabular}






Equal $\mathrm{AL}$ in the $\mathrm{HF}$ groups

Interestingly, this study showed an equal $\mathrm{AL}$ across the whole spectrum of LVEF. Accordingly, patients with HFpEF were not diagnosed more frequently with COPD than the other two groups. This is in line with previous research, ${ }^{17}$ but not all. ${ }^{18}$ This variation in COPD prevalence in different HF groups may be influenced by different factors including patient selection but importantly also misdiagnosis of COPD. ${ }^{19}$ The diagnosis of COPD was often based solely on medical history without further testing and not systematically applying spirometry. ${ }^{7}$ Diagnosing COPD was also frequently done by performing spirometry in hospitalised patients, where fluid overload may have influenced results significantly. ${ }^{7}$ It is important to keep these limitations in mind when interpreting results regarding prevalence of COPD in the different groups of HF. ${ }^{7}$

Possible differences with other studies may also be related to presence of risk factors other than HF, for example, smoking is linked to $\mathrm{AL}$ and higher risk of $\mathrm{COPD}^{20}$ and it is obvious that extent of smoking may influence results. Interestingly, left ventricular hypertrophy $(\mathrm{LVH})$ may be also related to $\mathrm{FEV}_{1} \cdot{ }^{21}$ The authors posed the hypothesis that COPD may influence LV structure. Patients known with COPD experience hyperinflation, an increase of intrathoracic pressure and a decrease of intrathoracic volume. In this cascade of events, the pulmonary vascular pressure will increase. All this will decrease the left ventricular end diastolic diameter and a reduction in preload. In this process, patients might develop a 'microcor', possibly resulting in chronic inflammation and pronounced activation of the renin-angiotensin-aldosterone system, ${ }^{22}$ which may result in paradoxical LVH. This study confirms these results, yet the order of this cascade is not certain. After all, not all patients with COPD but only the patients with severe emphysema develop a microcor. ${ }^{23}$ In addition, decreased preload was found to reduce the chance to develop LVH. ${ }^{24}$ Given the fact that only very few patients of the HF population of this study had severe COPD, it is much more likely that changes of the heart and its function may have influenced lung function abnormalities.

\section{Equal DLCO in all HF groups}

The present study found similar limitation in DLCO in the three HF groups. Previous research noticed a lower DLCO in patients with HF compared with healthy subjects. ${ }^{25}$ Thus, HF may play an important role, among other factors, in the reduction of DLCO. In patients with HF performing exercise, pulmonary capillary hydrostatic pressure may increase, lymphatic fluid removal can decrease and a reduction of active transport through sodium channels might develop. In this manner, pulmonary fluid overload is produced and the conductance of the alveolar capillary membrane may decrease. If the decrease in alveolar membrane conductance is greater than the increase in pulmonary capillary blood volume, DLCO decreases. ${ }^{26}$ The latter occurs if the pulmonary capillary blood flow decreases, for example, during the pathogenesis of pulmonary hypertension. ${ }^{27}$ Thus, a consequence of HF can be a decreased gas exchange at rest and a lower increase during exercise as compared with healthy subjects. ${ }^{28}$ Interestingly, diffusion capacity was impaired in $55 \%$ of the patients independently of the LVEF and showed a correlation with RV function, suggesting that such alterations may be present even in stable HF patients without evidence of congestion. Importantly, AL influenced diffusion capacity as expected, but it was also significantly reduced in HF patients without AL, independently of the LVEF. The significant though relatively weak correlation of DLCO with NT-proBNP as a measure of severity of HF further supports a direct influence of 
HF on gas exchange. Although these findings require confirmation in a larger cohort, they may be clinically meaningful as they might explain some physical limitation in patients with HF and can be of prognostic significance. ${ }^{1029} 30$ In addition, the influence of HF treatment should be investigated in this regard.

Obviously, HF is not the only factor influencing DLCO. Among the less well-known factors, male gender and increased BMI may influence DLCO. ${ }^{31-33}$ The influence of gender may be explained by the higher prevalence of smoking in men compared with women. The findings of the correlation higher BMI for a lower DLCO has been discussed in a previous study that found an alveolar enlargement and a decrease of ratio alveolar surface area-to-lung volume in obese rats. ${ }^{32}$

\section{A comparable RV function in the three HF groups}

A comparable RV function (i.e., TAPSE/PASP) was found in the three HF groups. The equal distribution of patients with COPD and pulmonary hypertension in all groups may have contributed to this, but this influence seems to be limited. This is in line with previous research that found similar RV function (i.e., TAPSE) in patients with non-severe COPD compared with healthy patients. ${ }^{3}$ Thus, LV failure in addition to the underlying cardiac pathology possibly affecting both ventricles is a much more important cause of RV failure due to first passive increase in pulmonary pressure, which may further result in an increase in pulmonary vascular resistance in some patients. ${ }^{34}$ The findings of the present study of correlations of TAPSE/PASP with NT-proBNP and pulmonary pressure supports this notion. The correlation between reduced systolic function of the LV and the RV may vary depending on the population included and needs to be better studied to determine the precise underlying cause of RV dysfunction in patients with HF. This may include invasive

\section{Key messages}

What is already known about this subject?

The link between heart failure (HF) and pulmonary abnormalities is already known. The relationship between the right ventricular function and pulmonary abnormalities is less clear. This relationship is not yet investigated in the three recent defined groups of HF (HF with reduced ejection fraction, $\mathrm{HF}$ with midrange ejection fraction (HFmrEF) and $\mathrm{HF}$ with preserved ejection fraction).

\section{What does this study add?}

Using a stable group of patients with $\mathrm{HF}$, a measurement of gas exchange in the lungs and the most accurate echocardiographic technique to estimate right ventricular function (eg, tricuspid annular plane systolic excursion/pulmonary artery systolic pressure), this study demonstrates accurate and state-of-theart information concerning the lungs and heart of the involved patients.

Another new element in this study is that lung abnormalities and right ventricular function are not influenced by left ventricular ejection fraction (LVEF). Thus, the pulmonary abnormalities and their frequencies are equal in the three groups.

How might this impact on clinical practice? This study emphasises the importance to perform extra investigation of the lungs in stable patients with $\mathrm{HF}$ independently of the LVEF. This is also applicable in the newly proposed and less investigated group of HFmrEF. measurements to better define these different factors potentially influencing the RV. The well-known association between RV dysfunction and poor outcome supports this need. ${ }^{11}$ Our results suggest that this need is not dependent on LVEF.

\section{Study strength and limitations}

The strengths of this study are the comprehensive patient characterisation regarding comorbidities, cause of HF, symptoms, lung function abnormalities and echocardiography. It is based on a representative group of stable, chronic HF patients subdivided according to the most recent ESC guidelines using a uniformed cut-off regarding LVEF. The measurements of pulmonary function and echocardiography occurred systematically according to state-of-the-art practice. Accordingly, the determination of HF and functional limitations of the lungs are accurate.

The main limitations are the relatively limited sample size, the single-centre setting, missing items for some of the variables and lack of invasive measurements and histological data. Therefore, it is possible that some differences between the three groups based on LVEF may become significant in a larger population. Still, the clinical consequences that lung function testing should be considered in all patients irrespectively of LVEF would not change. NT-proBNP values were measured in only 123 patients because this was not compulsory per protocol, and only values from study visits were used. Similarly, E/e' was not obtained in all patients as we used clinically indicated echocardiograms if done within 6 months, and these values were available in all. Systolic pulmonary pressure was only obtained if clear signal of tricuspid regurgitation was available, which was not the case in all patients. Still, patients without these measurements did not differ from the others (data not shown), which makes it rather unlikely that these missing data influenced the outcome of our study significantly. Given the stable situation and the fact that patients were well compensated, NT-proBNP and E/e' levels were on average relatively low and results might differ in patients with more advanced HF. Because of the lack of invasive measurements, it is impossible to precisely explore the pathophysiology of lung function abnormalities, pulmonary vascular disease, pulmonary hypertension and diagnosis of RV dysfunction.

Based on the power of this small study population and the amount of missing variables, no multivariable regression analysis was performed on this study population to investigate other predisposing factors for cardiopulmonary abnormalities. Future research on a large scale can be interesting to investigate these factors and potential causal links between them. Finally, we did not investigate any influence of treatment of HF on pulmonary abnormalities and vice versa. Such intervention studies may help to improve treatment of such patients and may improve understanding of pathophysiological interaction between heart and lungs.

\section{CONCLUSIONS}

Pulmonary function in terms of both diffusion capacity and airway limitation was reduced in a significant proportion of patients in this stable outpatient HF population, with no differences between HFpEF, HFmrEF and HFrEF. The reduction of diffusion capacity was even seen in a substantial proportion of patients who did not have airway limitations. Also, RV function was similarly affected in all three groups. The findings of this study underline the importance of identifying lung function abnormalities in stable patients with HF independently of the type of HF. 
Contributors $\mathrm{H}-\mathrm{PB}-\mathrm{LR}, \mathrm{CK}, \mathrm{VvE}, \mathrm{SB}$ and WR planned this research by inventing the research question. $\mathrm{H}-\mathrm{PB}-\mathrm{LR}$ and $\mathrm{SB}$ planned this research by making the set-up of this study. H-PB-LR, CK, VvE, JB, NU-L conducted this research by giving critical revisions. H-PB-LR, SB and WR reported the research by writing the manuscript. $H-P B-L R$ and WR made the figures. WR submitted the research. H-PB-LR is the guarantor.

Funding This study was funded by GlaxoSmithKline Pharma.

Competing interests We report grants from GSK-Pharma during the conduct of this study.

Patient consent Obtained.

Ethics approval The local medical ethics committee approved this study (METC 12-2-005 (NL39223.068.12))

Provenance and peer review Not commissioned; externally peer reviewed.

(c) Article author(s) (or their employer(s) unless otherwise stated in the text of the article) 2017. All rights reserved. No commercial use is permitted unless otherwise expressly granted.

\section{REFERENCES}

1 Ponikowski P, Voors AA, Anker SD, et al. 2016 ESC Guidelines for the diagnosis and treatment of acute and chronic heart failure: The Task Force for the diagnosis and treatment of acute and chronic heart failure of the European Society of Cardiology (ESC). Developed with the special contribution of the Heart Failure Association (HFA) of the ESC. Eur J Heart Fail 2016;18:891-975.

2 Olson TP, Johnson BD, Borlaug BA. Impaired pulmonary diffusion in Heart failure with preserved Ejection Fraction. JACC Heart Fail 2016;4:490-8.

3 Cuttica MJ, Shah SJ, Rosenberg SR, et al. Right heart structural changes are independently associated with exercise capacity in non-severe COPD. PLoS One 2011:6:e29069.

4 Miniño AM, Xu J, Kochanek KD. Deaths: preliminary data for 2008. Natl Vital Stat Rep 2010:59:1-52.

5 Guazzi M, Pontone G, Brambilla R, et al. Alveolar_capillary membrane gas conductance: a novel prognostic Indicator in chronic heart failure. Eur Heart J 2002;23:467-76.

6 Hardie JA, Buist AS, Vollmer WM, et al. Risk of over-diagnosis of COPD in asymptomatic elderly never-smokers. Eur Respir J 2002:20:1117-22.

7 Hawkins NM, Petrie MC, Jhund PS, et al. Heart failure and chronic obstructive pulmonary disease: diagnostic pitfalls and epidemiology. Eur J Heart Fail 2009;11:130-9.

8 Kapoor JR, Kapoor R, Ju C, et al. Precipitating clinical factors, Heart Failure Characterization, and Outcomes in Patients Hospitalized With Heart Failure With Reduced, Borderline, and Preserved Ejection Fraction. JACC Heart Fail 2016;4:464-72.

9 Hilde JM, Skjørten I, Grøtta OJ, et al. Right ventricular dysfunction and remodeling in chronic obstructive pulmonary disease without pulmonary hypertension. J Am Coll Cardiol 2013;62:1103-11.

10 Guazzi M, Bandera F, Pelissero G, et al. Tricuspid annular plane systolic excursion and pulmonary arterial systolic pressure relationship in heart failure: an index of right ventricular contractile function and prognosis. Am J Physiol Heart Circ Physiol 2013:305:H1373-81.

11 Meyer P, Filippatos GS, Ahmed Ml, et al. Effects of right ventricular ejection fraction on outcomes in chronic systolic heart failure. Circulation 2010;121:252-8.

12 McMurray JJ, Adamopoulos S, Anker SD, et al. ESC guidelines for the diagnosis and treatment of acute and chronic heart failure 2012: the Task Force for the diagnosis and treatment of acute and chronic Heart failure 2012 of the european Society of Cardiology. developed in collaboration with the Heart failure association (HFA) of the ESC. Eur J Heart Fail 2012;14:803-69.
13 Souza R, Fernandes CJ, Hoeper MM. Carbon Monoxide diffusing capacity and the complexity of diagnosis in pulmonary arterial hypertension. Eur Respir J 2014;43:963-5.

14 Vestbo J, Hurd SS, Agustí AG, et al. Global strategy for the diagnosis, management, and prevention of chronic obstructive pulmonary disease: gold executive summary. Am J Respir Crit Care Med 2013:187:347-65.

15 Cotes JE, Chinn DJ, Quanjer PH, et al. Standardization of the measurement of transfer factor (diffusing capacity). Report Working Party standardization of lung function tests, European Community for Steel and Coal. Official Statement of the european respiratory Society. Eur Respir J Supp/ 1993;16:41-52.

16 Lang RM, Badano LP, Mor-Avi V, et al. Recommendations for cardiac chamber quantification by echocardiography in adults: an update from the American Society of Echocardiography and the European Association of Cardiovascular Imaging. Eur Heart J Cardiovasc Imaging 2015;16:233-71.

17 Ahmed A, Roseman JM, Duxbury AS, et al. Correlates and outcomes of preserved left ventricular systolic function among older adults hospitalized with heart failure. $A m$ Heart J 2002;144:365-72.

18 Berry C, Hogg K, Norrie J, et al. Heart failure with preserved left ventricular systolic function: a hospital cohort study. Heart 2005:91:907-13.

19 Chapman KR, Mannino DM, Soriano JB, et al. Epidemiology and costs of chronic obstructive pulmonary disease. Eur Respir J 2006;27:188-207.

20 Mathers $C D$, Loncar D. Projections of global mortality and burden of disease from 2002 to 2030. PLOS Med 2006:3:e442.

21 Guazzi M, Villani S, Generati G, et al. Right ventricular contractile reserve and Pulmonary circulation uncoupling during exercise challenge in Heart failure: pathophysiology and clinical phenotypes. JACC Heart Fail 2016;4:625-35.

22 Pelà G, Li Calzi M, Pinelli S, et al. Left ventricular structure and remodeling in patients with COPD. Int J Chron Obstruct Pulmon Dis 2016;11:1015-22.

23 Jörgensen K, Müller MF, Nel J, et al. Reduced intrathoracic blood volume and left and right ventricular dimensions in patients with severe emphysema: an MRI study. Chest 2007;131:1050-7.

24 Voigt JU, Lindenmeier G, Werner D, et al. Strain rate imaging for the assessment of preload-dependent changes in regional left ventricular diastolic longitudinal function. J Am Soc Echocardiogr 2002;15:13-19.

25 Olson LJ, Snyder EM, Beck KC, et al. Reduced rate of alveolar-capillary recruitment and fall of pulmonary diffusing capacity during exercise in patients with heart failure. J Card Fail 2006;12:299-306.

26 Puri S, Dutka DP, Baker BL, et al. Acute saline infusion reduces alveolar-capillary membrane conductance and increases airflow obstruction in patients with left ventricular dysfunction. Circulation 1999:99:1190-6.

27 Johnson RL. Gas exchange efficiency in congestive heart failure II. Circulation 2001:103:916-8.

28 Puri S, Baker BL, Dutka DP, et al. Reduced alveolar-capillary membrane diffusing capacity in chronic heart failure. its pathophysiological relevance and relationship to exercise performance. Circulation 1995;91:2769-74.

29 Guazzi M, Naeije R, Arena R, et al. Echocardiography of right ventriculoarterial coupling combined with cardiopulmonary exercise testing to predict outcome in heart failure. Chest 2015;148:226-34.

30 Methvin AB, Owens AT, Emmi AG, et al. Ventilatory inefficiency reflects right ventricular dysfunction in systolic heart failure. Chest 2011;139:617-25.

31 Banks E, Joshy G, Weber MF, et al. Tobacco smoking and all-cause mortality in a large Australian cohort study: findings from a mature epidemic with current low smoking prevalence. BMC Med 2015;13:38

32 Inselman LS, Padilla-Burgos LB, Teichberg S, et al. Alveolar enlargement in obesityinduced hyperplastic lung growth. J Appl Physiol 1988;65:2291-6.

33 Zavorsky GS, Kim DJ, Sylvestre JL, et al. Alveolar-membrane diffusing capacity improves in the morbidly obese after bariatric surgery. Obes Surg 2008:18:256-63.

34 Guazzi M, Galiè N. Pulmonary hypertension in left heart disease. Eur Respir Rev 2012:21:338-46. 